Original Article

\title{
Does fatigue expose functional deficits in chronic lateral ankle sprain?
}

\author{
Aikaterini Karkatselou, PT, BSc ${ }^{1)^{*}}$, Panagiotis Gkrilias, PT, MSc, $\mathrm{PhD}^{1)}$, \\ ELIAS TSEPIS, PT, BSc, MSc, $\mathrm{PhD}^{1)}$
}

1) Department of Physiotherapy, University of Patras: Psaron 6, Myrtia, 25100, Aigio, Greece

\begin{abstract}
Purpose] The purpose of this study was to examine whether functionally induced fatigue affects balance and vertical jump performance more severely in individuals with a history of unilateral lateral ligament injury to the ankle joint. [Participants and Methods] Twenty-three participants volunteered for the study with the experimental group (EG $\mathrm{n}=12$ ) comprising injured participants with stability deficits and lack of physiotherapy treatment tested against healthy controls $(C G n=11)$. Balance was assessed via a single leg balance test with open eyes on a force platform. The Center-of-Pressure (COP) excursion on the anteroposterior (Y-axis) and the mediolateral (X-axis) were recorded. Additionally, maximal single-leg vertical jump height (VJH) was assessed on a specific platform. Ankle fatigue was induced through performing the modified Square-hop test until exhaustion. [Results] Patients exhibited significant interaction for Time X Group for the COP on the X-axis. COP-X which did not differ between groups, pre-fatigue, while post-fatigue, it increased significantly in the EG. VJH demonstrated significant pre-fatigue differences between groups, suggesting that deficits in this variable were evident without the need to fatigue the muscles involved. [Conclusion] Fatigue can be useful when balance is tested, since post-fatigue deficits, otherwise obscured in the baseline measurement, can become significant.

Key words: Ankle sprains, Fatigue, Balance deficits
\end{abstract}

(This article was submitted Jul. 28, 2019, and was accepted Oct. 17, 2019)

\section{INTRODUCTION}

Lateral ankle sprain (LAS) is the most common musculoskeletal injury in sports, especially in competitive sports including jumping and multi-directional changes ${ }^{1)}$. After multiple or severe ankle sprains, post-injury anatomic laxity as well as functional-dynamic instability are established in the affected joint. Muscle activity around the ankle joint plays an important part for the sensorimotor control and stability of the ankle ${ }^{2)}$. After a LAS, damage occurs not only to the ligament but also to the neuromuscular structures surrounding the ankle joint ${ }^{3)}$. Kinesthetic as well as joint position and force sense deficits are present in individuals with functional ankle instability resulting from damage to the muscle mechanoreceptors and subsequently impair an individual's ability to provide the necessary muscle force to stabilize the joint in dynamic conditions ${ }^{4}$. Neuromuscular control plays a major role in dynamic joint stability ${ }^{5)}$ and peripheral fatigue may impair this control and stability $^{6}$ via desensitizing muscle spindles and the afferent pathways to the central nervous system. Subsequently, this slows the response and the number of muscle fibers needed to handle ankle perturbations increasing the possibility for injury ${ }^{7)}$. A variety of fatigue inducing methods exist (REFS), however, it is important that this procedure is functional, simulating real life conditions. Single-leg hopping tests are widely used for functional performance testing ${ }^{8}$, and considering the aforementioned, when those tests are executed under fatigue, they could expose functional deficits otherwise concealed. The purpose of this study was to examine the effect of functionally induced fatigue on balance and vertical jump performance in individuals with a history of unilateral ligament injury to the ankle joint.

*Corresponding author. Aikaterini Karkatselou (E-mail: karkatse@gmail.com)

(C)2020 The Society of Physical Therapy Science. Published by IPEC Inc.

(c) (1) $\odot$ This is an open-access article distributed under the terms of the Creative Commons Attribution Non-Commercial No Derivatives cc) (by-nc-nd) License. (CC-BY-NC-ND 4.0: https://creativecommons.org/licenses/by-nc-nd/4.0/) 


\section{PARTICIPANTS AND METHODS}

A total of twenty-three participants ( 8 males, 15 females) volunteered for this study (age: $22.13 \pm 2.94$ years, height: $168 \pm 8 \mathrm{~cm}$, body mass: $67.33 \pm 9.70 \mathrm{~kg}$, right leg length: $85.57 \pm 5 \mathrm{~cm}$, left leg length: $85.45 \pm 4.91 \mathrm{~cm})$. The experimental group (EG $n=12$ ) comprising injured participants, was tested against healthy controls $(\mathrm{CG} n=11)$. The injured participants had history of repeated unilateral ankle sprains and declared they had functionally unstable ankle. Patients with a history of major trauma to the ankle joint, were excluded from the study. At the time of testing, the experimental group demonstrated moderate ankle instability and all participants were pain free. The healthy controls had never experienced ankle sprain in any limb. The experimental design of this study was approved (41480/30-11-2017) by the Ethics Committee of the School of Health Sciences (formerly in the Technological Educational Institute of Western Greece, now incorporated at the University of Patras) and testing took place in the Human Assessment and Rehabilitation Laboratory of the University of Patras, Greece. Prior to testing, all participants were initially briefed on the experimental procedure, signed a consent form and completed questionnaires detailing the history of ligament injuries, the functional instability of ankle ligament and foot dominance (WFQr) ${ }^{9}$. The injured limb from the EG and the dominant limb from the CG were tested, since it has been shown that athletes appear to balance better in the dominant leg ${ }^{10)}$. The experimental process included 3 sessions intercepted by 3-7 days. The first session included the familiarization of the participant with the tasks and the measurement of maximal neuromuscular ability until exhaustion. During the first session, each participant's baseline maximal number of single-leg plantar to dorsiflexion cycles was recorded until exhaustion, performed on a $10 \mathrm{~cm}$ step, with a pace of $80 \mathrm{bpm}$ kept by a metronome ${ }^{8)}$. The number of repetitions (full cycles) recorded for each limb was used to define the state of fatigue. Counting stopped when the participant could no longer elevate the heel from the ground. The next 2 sessions included the main tasks, one for each limb. Balance was assessed via a single-leg balance test with open eyes on a force platform (AMTI BP400600-OP force platform \& AMTI MiniAmp MSA-6) for $20 \mathrm{sec}^{11)}$. During this test, each participant was standing in single-leg stance with the foot on the platform, arms akimbo and eyes on a target 4 meters away, at eye level. The task ended when the participant completed 20 seconds without loss of balance, with the procedure repeated once more. The Center-of-Pressure (COP) excursion on the anteroposterior (Y-axis), the mediolateral (X-axis) and ABS were recorded. Additionally, maximal single-leg vertical jump height (VJH) was assessed on a specific platform (ChronoJump BoscoSystem), where each participant was standing in single-leg stance with the foot on the platform, arms akimbo and motivated to jump as high as possible ${ }^{12)}$. The highest jump was recorded from 5 consecutive jumps. Ankle fatigue was induced through performing the modified Square-Hop test until exhaustion ${ }^{13}$, where a $40 \times 40 \mathrm{~cm}$ square was marked on the floor and participants were instructed to hop in and out of the square completely around the square, as fast as possible for 30 seconds followed by 30 seconds of rest, for 5 times. After this procedure, the level of fatigue was assessed with the Modified Borg Scale. In case of 8/10 or higher, the fatigue protocol was completed and endurance until exhaustion was tested as in the baseline heel-lift session. Adequate fatigue was confirmed when the number of repetitions was reduced by at least $50 \%$ from the baseline testing and the participants proceeded with the balance and VJH tasks in the same way and order. Two-way ANOVA, 2(Group) $\times 2$ (Time), was used for statistical analysis (SPSS v20).

\section{RESULTS}

Statistical analysis showed significant interaction for Time $\times$ Group on the mediolateral axis $(\mathrm{p}=0.050)$, but not on the anteroposterior axis $(\mathrm{p}=0.470)$. Post-hoc analysis showed that COP-X values increased significantly in the experimental group post-fatigue, while no change was noticed in the control group. Regarding the maximal single-leg vertical jump height, both groups were significantly influenced by fatigue (EG: $\mathrm{p}=0.001$, CG: $\mathrm{p}=0.000$ ). All results appear on Table 1 .

\section{DISCUSSION}

The purpose of the current study was to assess whether specific parameters of unilateral balance testing and vertical jump height were influenced more adversely in fatigued patients with chronic lateral ankle instability compared to controls. Regarding balance, patients exhibited greater post-fatigue deficits on the frontal plane while there was no significant effect on the anteroposterior plane. This might reveal an important functional deficit since, lateral ankle injuries occur while failing to control dynamic activities with mediolateral stress during landing. The EG showed higher post-fatigue mediolateral postural sway values compared to CG while there were no statistically significant pre-fatigue differences between groups. This indicates that performance under fatigue appears to reveal otherwise possibly concealed deficits, potentially influencing susceptibility for re-injury6). These findings appear in line with previous studies ${ }^{4}$ which have shown that repetitive ankle sprains could lead to force sense deficits to the muscles responsible for the stability of the ankle ligament. Also, the lack of a proper physiotherapy program for our participants in the experimental group after every ankle ligament injury, could impair dynamic control and therefore, provoke ankle instability. This stability deficit was caused because of the history of ligament injury in that particular group and the lack of physiotherapy treatment after the injury ${ }^{3)}$. The method of inducing muscle fatigue with the use of a simulated Square-Hop test is our proposal as an alternative, functional way to fatigue the ankle stabilizing muscles. The key finding of this study is that, repeated multidirectional jumps performed in a standardized man- 
Table 1. The results for 2 (Group) $\times 2$ (Time) ANOVA and Post-hoc analysis for Cop X, Cop Y and single leg vertical jump height (VJH)

\begin{tabular}{|c|c|c|c|c|c|c|c|c|c|c|c|}
\hline \multicolumn{2}{|c|}{ Parameter } & \multirow{2}{*}{$\begin{array}{l}\text { Pre-fatigue } \\
\text { Mean } \pm \text { SD }\end{array}$} & \multirow{2}{*}{$\begin{array}{l}\text { Post-fatigue } \\
\text { Mean } \pm \text { SD }\end{array}$} & \multicolumn{2}{|c|}{$\begin{array}{c}2(\text { Group }) \times 2(\text { Time }) \\
\text { ANOVA }\end{array}$} & \multicolumn{2}{|c|}{$\begin{array}{c}\text { Post-hoc analysis } \\
\text { within groups } \\
\text { (Repeated ANOVA) }\end{array}$} & \multicolumn{2}{|c|}{$\begin{array}{l}\text { Post-hoc control vs. } \\
1 \text { Leg Pre-fatigue }\end{array}$} & \multicolumn{2}{|c|}{$\begin{array}{l}\text { Post-hoc control vs. } \\
1 \text { leg Post fatigue }\end{array}$} \\
\hline & & & & F-value & $\begin{array}{l}\text { p-value } \\
\text { (Sig) }\end{array}$ & F-value & $\begin{array}{l}\text { p-value } \\
\text { (Sig) }\end{array}$ & F-value & $\begin{array}{l}\text { p-value } \\
\text { (Sig) }\end{array}$ & F-value & $\begin{array}{l}\text { p-value } \\
\text { (Sig) }\end{array}$ \\
\hline \multirow[t]{2}{*}{ Cop X } & $\mathrm{CG}$ & $0.012 \pm 0.007$ & $0.012 \pm 0.013$ & 4.329 & 0.05 & & & & 0.167 & & 0.015 \\
\hline & EG & & & & & & & & & & \\
\hline \multirow[t]{2}{*}{ Cop Y } & $\mathrm{CG}$ & $0.021 \pm$ & 0.030 & 0.543 & 0.470 & 1.120 & 0.315 & 0.386 & 0.541 & 1.024 & 0.323 \\
\hline & EG & & 0.044 & & & & & & & & \\
\hline \multirow[t]{2}{*}{ 1LegVJH } & CG & $14.57 \pm 2.82$ & $12.92 \pm 3.28$ & 5.374 & 0.031 & 25.649 & 0.000 & 18.048 & 0.000 & 11.223 & 0.003 \\
\hline & EG & $8.96 \pm 3.44$ & $8.16 \pm 3.51$ & & & 19.119 & 0.001 & & & & \\
\hline
\end{tabular}

Cop X: Center of pressure in mediolateral axis; Cop Y: Center of pressure in anteroposterior axis; 1Leg VJH: Maximal single-leg vertical jump height; CG: Control group; EG: Experimental group.

ner, caused significant balance and VJH impairments in patients with a history of lateral ankle sprain. Single leg balance is a less dynamic task, thus functional post-injury deficits might have been more easily obscured. However, those deficits were exposed when fatigue was introduced. This finding could be clinically useful, suggesting that fatigue can be important during testing. The frontal plane is mainly involved in lateral ligamentous injury to the ankle. In addition, balance on this plane is naturally more unstable, compared to the A-P plane. Thus, stability deficits in chronic ankle instability are more prone to be exposed on the less stable plane. The study was limited by the fact that joint laxity was not assessed via imaging techniques. However, a significant number of similar studies are based on self-reported questionnaires and clinical tests ${ }^{4,13)}$. Additionally, the experimental group consisted of young individuals and long-term post-injury functional adaptations might not have been established. Aging with a history of joint injury could bear more obvious adaptations. Future EMG investigation on the effects of functional fatigue on functional testing could provide an in-depth explanation of our findings. In conclusion, inducing fatigue before balance testing should be considered, since, in the current study, post-fatigue deficits, not evident in the baseline assessment, became significant.

Conflict of interest

None.

\section{REFERENCES}

1) Doherty C, Delahunt E, Caulfield B, et al.: The incidence and prevalence of ankle sprain injury: a systematic review and meta-analysis of prospective epidemiological studies. Sports Med, 2014, 44: 123-140. [Medline] [CrossRef]

2) Lin CF, Chen CY, Lin CW: Dynamic ankle control in athletes with ankle instability during sports maneuvers. Am J Sports Med, 2011, 39: 2007-2015. [Medline] [CrossRef]

3) Hertel J: Functional instability following lateral ankle sprain. Sports Med, 2000, 29: 361-371. [Medline] [CrossRef]

4) Docherty CL, Arnold BL: Force sense deficits in functionally unstable ankles. J Orthop Res, 2008, 26: 1489-1493. [Medline] [CrossRef]

5) Riemann BL, Lephart SM: The sensorimotor system II: the role of proprioception in motor control and functional joint stability. J Athl Train, 2002 , 37: $80-84$. [Medline]

6) Forestier N, Teasdale N, Nougier V: Alteration of the position sense at the ankle induced by muscular fatigue in humans. Med Sci Sports Exerc, 2002, 34: 117-122. [Medline] [CrossRef]

7) Yalfani A, Gandomi F, Abbasi H: The effect of fatigue on the ankle and knee proprioception and dynamic control of posture. Inter J Sports Sci Fit, 2013, 3: 221-229.

8) Augustsson J, Thomeé R, Lindén C, et al.: Single-leg hop testing following fatiguing exercise: reliability and biomechanical analysis. Scand J Med Sci Sports, 2006, 16: 111-120. [Medline] [CrossRef]

9) Kapreli E, Athanasopoulos S, Stavridis I, et al.: Waterloo Footedness Questionnaire (WFQR): cross-cultured adaptation and psychometric properties of Greek version. Physiotherapy, 2015, 101: 721. [CrossRef]

10) Gkrilias P, Zavvos A, Fousekis K, et al.: Dynamic balance asymmetries in pre-season injury-prevention screening in healthy young soccer players using the Modified Star Excursion Balance Test-a pilot study. J Phys Ther Sci, 2018, 30: 1141-1144. [Medline] [CrossRef]

11) Bisson EJ, Chopra S, Azzi E, et al.: Acute effects of fatigue of the plantarflexor muscles on different postural tasks. Gait Posture, 2010, 32: 482-486. [Medline] [CrossRef]

12) Kockum B, Heijne AI: Hop performance and leg muscle power in athletes: reliability of a test battery. Phys Ther Sport, 2015, 16: 222-227. [Medline] [CrossRef]

13) Caffrey E, Docherty CL, Schrader J, et al.: The ability of 4 single-limb hopping tests to detect functional performance deficits in individuals with functional ankle instability. J Orthop Sports Phys Ther, 2009, 39: 799-806. [Medline] [CrossRef] 